\title{
Factor personal e institucional en el desarrollo del "cuidado humano de ayuda confianza", de la teoria de Jean Watson en los enfermeros del servicio de medicina 3B del Hospital Nacional Guillermo Almenara Irigoyen, 2013
}

\author{
Carolina Sebastián Llancari ${ }^{1, a, b}$, Doris Velásquez Carranza ${ }^{2,,, d}$
}

\begin{abstract}
RESUMEN
La teoría del cuidado humano de Watson sostiene que ante el riesgo de deshumanización en el cuidado del paciente, se hace necesario el rescate del aspecto humano por parte de los profesionales de enfermería. Objetivos: determinar el factor personal e institucional en el desarrollo del "cuidado humano de ayuda-confianza", de la teoría de Jean Watson en los enfermeros en el Servicio de Medicina 3B del HNGAI. Material y métodos: estudio descriptivo de corte transversal. Población conformada por el total de enfermeros (35), que cumplieron los criterios de selección. Instrumento fue elaborado por las autoras. Las técnicas de recolección de datos fueron la encuesta y la observación; los instrumentos, un cuestionario estructurado auto administrado y una guía de observación. Los datos recolectados procesados utilizando el paquete estadístico de STATAv 12. Resultados: la frecuencia del factor personal (motivación, satisfacción profesional y relaciones interpersonales) está presente en un $60 \%$ y el factor institucional (capacitación) en un $80 \%$. De las dimensiones observadas, se obtuvo la comunicación eficaz (97,14\%), coherencia y acogida no posesiva $(88,57 \%)$ y empatía $(77,14 \%)$. Conclusiones: el factor personal e institucional están siempre presentes en el desarrollo del "Cuidado humano de ayudaconfianza" y en los enfermeros observados se encontró que se brinda el cuidado aplicando el cuarto factor según sus dimensiones.
\end{abstract}

PALABRAS CLAVE: Relación enfermera-paciente, humanización de la atención.

\section{Personal and institutional development of "human care support trust" factor, the theory of Jean Watson in nurses at the 3B Medical Service of the Hospital Nacional Guillermo Almenara Irigoyen, 2013}

\section{SUMMARY}

The theory of human caring Watson argues that given the risk of dehumanization in patient care, rescue the human aspect by nurses is necessary. Objectives: To determine the personal and institutional factor in the development of "human care aid-confidence," the theory of Jean Watson in nurses in the Department of Medicine HNGAI 3B. Material and Methods: A descriptive cross-sectional study. Consists of the total population of nurses (35), who met the selection criteria. The techniques of data collection were the surve $y$ and observation;

Hospital Guillermo Almenara Irigoyen, EsSalud. Lima, Perú.

Facultad de Enfermería, Universidad Peruana Cayetano Heredia. Lima, Perú.

Licenciada en Enfermería ; ${ }^{b}$ Enfermera Asistencial ; ${ }^{\mathrm{c}}$ Magister en Enfermería ; ${ }^{\mathrm{d}}$ Docente Asociada 
instruments, structured self-administered questionnaire and an observation guide. The collected data processed using the statistical package STATA v 12. Results: The frequency of the personal factor (motivation, satisfaction and professional relationships) is present in $60 \%$ and institutional factor (training) by $80 \%$. From the observed dimensions, effective communication (97.14\%), coherence and hostnonpossessive (88.57\%) and empathy (77.14\%) were obtained. Conclusions: The personal and institutional factors are always present in the development of "human care aid-confidence" and in the observed nurses found that care is provided by applying the fourth factor according to their dimensions.

KEYWORDS: Nurse-patient relationship, humanization of care.

\section{INTRODUCCIÓN}

La "Teoría del cuidado humano", sostiene que ante el riesgo de deshumanización en el cuidado del paciente, a causa de la gran reestructuración administrativa de la mayoría de los sistemas de cuidado de salud en el mundo, se hace necesario el rescate del aspecto humano, espiritual y transpersonal en la práctica clínica, administrativa, educativa y de investigación por parte de los profesionales de enfermería.

Una relación de ayuda-confianza fomenta y acepta la expresión tanto de los sentimientos positivos como de los negativos. Implica coherencia, empatía, acogida no posesiva y comunicación eficaz con los enfermeros, para brindar cuidado humano, individualizado, integral y ético. Este tipo de cuidado debe reflejarse en la gestión del cuidado que planifiquen, ejecuten y evalúen los profesionales de enfermería en cada servicio donde presten cuidados de calidad; sin embargo, existen factores (personales e institucionales) que influyen en ésta relación de manera positiva o negativa, por lo cual evidenciarlos puede contribuir a una mejor interacción entre la enfermera-paciente (1).

La enfermera es la persona que establece las relaciones afectivas y de esperanza más estrechas con los pacientes. Estas relaciones que se dan en el proceso de atención, son muy complejas y están influenciadas por múltiples situaciones y factores. Por esto, los cuidados de enfermería suponen una confrontación positiva y activa entre la enfermera, el equipo de salud y los pacientes.

Cada vez es más frecuente ver a los pacientes en contacto con dispositivos de cuidado que dificultan la interacción humana y a los enfermeros (as) involucradas con el uso de la tecnología que los vuelve rutinarios y genere en ellos actitudes que los predisponen al alejamiento de los pacientes; sin embargo, los enfermeros (as) no deben dejarse confundir por esta falsa idea, porque la interacción humana es inherente a la enfermería. La interacción no puede ser reemplazada ya que es el medio por el cual los enfermeros (as) llevan a cabo el propósito de la Enfermería (2).

Por tanto los cuidados de enfermería no pueden realizarse sin establecer relaciones con los pacientes, es decir, no es posible cuidar sin formar vínculo alguno con los pacientes. Esta relación es un acto continuo y dinámico, es un vínculo interpersonal permanente en el que el proceso de comunicación está siempre presente. Así, la esencia del cuidado descansa en el diálogo, la comunicación y la interrelación entre el enfermero, el paciente o sus familiares (3).

La base de la teoría de Watson es la práctica enfermera de los diez factores del cuidado (4), cada uno de ellos tiene un componente fenomenológico relativo a los individuos implicados en la relación que abarca la enfermería; el desarrollo de una relación de cuidado humano de ayuda-confianza entre la enfermera y el paciente es crucial para el cuidado transpersonal (5). En nuestra realidad, lo que experimentamos es una falta del cuidado humano puesto que nuestro ejercicio de enfermería está sostenido sólo en las prácticas médicas de tratamiento lo cual significa que no estamos cuidando. Los enfermeros (as) muchas veces no adoptan formas conductuales que permitan empatía e identificación con el paciente, por ello el enfermero debe propiciar formas apropiadas de comunicación con el paciente que presenta dificultades para relacionarse $o$ que habla otro idioma o dialecto, debe velar por el derecho del paciente a la comunicación y mantener los lazos afectivos con su familia y con el entorno.

En el Servicio de Medicina 3B (Este y Oeste) del Hospital Nacional Guillermo Almenara Irigoyen en Lima, Perú, los enfermeros (as) perciben una gran demanda de ingresos de pacientes transferidos del Servicio de Emergencia, adultos mayores, dependientes, considerados de alto riesgo por la involución de las funciones de los diferentes aparatos y sistemas productos de la edad, 
sumado a ello el deterioro del estado de conciencia siendo un alto factor de riesgo para producir lesiones sobre agregadas (autolesiones, caídas), llevando todo ello a un deterioro del desarrollo de la relación de cuidado humano de ayuda-confianza que puedan brindar los enfermeros (as) a los pacientes y/o familiares.

El objetivo del presente trabajo fue dar a conocer el factor personal e institucional en el desarrollo del cuarto factor "Cuidado humano de ayuda confianza", en el quehacer enfermero basado en la Teoría de Jean Watson.

\section{MATERIAL Y METODOS}

El estudio corresponde a una investigación de enfoque cuantitativo, tipo de estudio descriptivo y de corte transversal.

La población fue de 35 enfermeros (total de enfermeros asistenciales) que laboran en el Servicio de Medicina 3B (Este y Oeste) del Hospital Nacional Guillermo Almenara Irigoyen. El estudio se realizó con toda la población quienes cumplen los criterios de selección. Una vez elaborado los instrumentos, previa validación y confiabilidad y aprobado por el Comité de Ética de la Universidad Peruana Cayetano Heredia y contando con la autorización de la Oficina Ejecutiva de apoyo a la Investigación y Docencia Especializada del Hospital Nacional Guillermo Almenara Irigoyen, la Jefatura del Departamento de Enfermería y la aprobación de las enfermeras jefas del Servicio de Medicina 3B (Este y Oeste) se procedió a la recolección de datos en dos etapas:

\section{$1^{\circ}$ Etapa: Etapa de la Encuesta}

1. Se captó a todas las enfermeras (os) el día de la reunión mensual de carácter obligatorio de servicio (Este y Oeste), llevándose a cabo en dos fechas, en el cual se brindó toda la información correspondiente sobre el estudio de investigación e instrumentos a aplicar.

2. En ambas fechas, se seleccionó del total de enfermeros (as) a aquellas que cumplían con los criterios de selección, contando con el total de enfermeros (as) para el estudio.

3. Se les solicitó a los enfermeros (as) que deseaban participar en forma voluntaria, la firma del consentimiento informado.

4. Por último, se aplicó la encuesta a todos los participantes del estudio, en un tiempo de 30 minutos.

\section{$2^{\circ}$ Etapa: Etapa de la observación}

1. Se verificó de la lista de enfermeros (as) participantes el chec que corresponde haber firmado el consentimiento informado y por consecuente respondido la encuesta.

2. Se observó el desarrollo de las actividades del personal enfermero considerando los turnos de seis horas, logrando aplicar la guía, tomando en cuenta dos grupos de guardia que estarán conformados por tres enfermeros (as) por cada semana, en los sectores este y oeste.

3. La guía de observación se aplicó por única vez a un total de 6 enfermeros por semana, estos formaban parte de dos grupos de guardia diferentes (tres enfermeros por equipo), de esta manera se buscó que no haya un recuerdo por parte de los participantes que puedan alterar la información (sesgos).

\section{RESULTADOS}

En los resultados encontrados se pudo observar que un $88,57 \%$ de los participantes fueron de género femenino y licenciados de enfermería, un $71,43 \%$ eran casados y solo trabajaban en la institución.

El $60 \%$ de los enfermeros consideran que la frecuencia del factor personal está basado en la motivación, satisfacción profesional y relaciones interpersonales encontrándose éste siempre presente para que se desarrolle el cuidado humano de ayuda-confianza enfermera-paciente.

Por otro lado, el factor institucional que siempre se presenta con mayor frecuencia para el desarrollo del cuidado humano de ayuda-confianza son la capacitación con un $80 \%$ y el reconocimiento laboral con un $68,6 \%$.

De esta manera, se encontró que con respecto a las dimensiones estudiadas del cuidado humano ayudaconfianza, todas se encuentran en un alto porcentaje, siendo la comunicación eficaz la de mayor frecuencia 97,14\% (tabla 3).

\section{DISCUSION}

En cuanto al factor personal, se puede decir que es el conjunto de elementos inherentes y propios de los individuos reflejados como valores, actitudes, creencias, carácter, temperamento, que se van consolidando en el perfil de la personalidad del individuo, marcando así la diferencia entre los mismos, cuya característica es la superación y realización personal.

En relación al factor personal, la población de estudio, según sus características sociodemográficas, estuvo conformada por 35 enfermeros (as), estando el 60\% entre 
los 44 a 63 años de edad, un 88,57\% de sexo femenino, $71,43 \%$ eran casados.

Los años de experiencia laboral predominante fue de $42,86 \%$ comprendida entre los 3 a 15 años, con un nivel de estudios de postgrado de $57,14 \%$ en la población de estudio y un $71,43 \%$ de los mismos que indicó que laboraban en una sola entidad de salud (tabla 1).

Así mismo, respecto a la frecuencia del factor personal de los enfermeros para el desarrollo del "Cuidado humano de ayuda-confianza" (Tabla 1); el 60\% de los enfermeros, considera que este factor está basado en la motivación, satisfacción personal y relaciones interpersonales; estos resultados son importantes ya que son factores propios de los enfermeros, los cuales pueden ser analizados y reforzados, generando actitudes reflexivas respecto a la mejora del cuidado humano; así mismo recordemos que Watson afirma que se debe otorgar especial interés a las circunstancias personales de los enfermeros para que las interacciones sean eficaces y brinden los mejores resultados.

Estos datos son similares con los resultados encontrados en el estudio "Factores que Influyen en el profesional de enfermería para la aplicación de los modelos y teorías de enfermería en el cuidado del usuario realizado por Armas, en la Microred de Chosica y Chaclacayo, Lima, donde se obtuvo que estos factores superan el $50 \%$, resaltando los conocimientos adquiridos en el pregrado, la motivación y el interés en la actualización para participar en cursos de capacitación (6).

De igual manera Martins y col., en su estudio "Resignificando la humanización desde el cuidado en el curso del vivir humano", propone un cuidado basado en la ética, valores humanos y siendo necesario el cambio de comportamiento, para ello es necesario buena voluntad, ser interiorizado para alcanzar los mejores niveles de calidad y de humanización de los servicios.

Tabla 1. Frecuencia del factor personal del enfermero para el desarrollo del "Cuidado humano de ayuda-confianza".

\begin{tabular}{lcccc}
\hline Factor personal & Siempre & \multicolumn{1}{l}{ A veces } & \multicolumn{1}{l}{ Nunca } & \multicolumn{1}{c}{ TOTAL } \\
\hline Motivación & $21(60 \%)$ & $9(25,7 \%)$ & $5(14,3 \%)$ & $35(100 \%)$ \\
Satisfacción Profesional & $21(60 \%)$ & $13(37,1 \%)$ & $1(2,9 \%)$ & $35(100 \%)$ \\
Relaciones interpersonales & $21(60 \%)$ & $13(37,1 \%)$ & $1(2,9 \%)$ & $35(100 \%)$ \\
\hline
\end{tabular}

Tabla 2. Frecuencia del factor institucional para el para el desarrollo del "Cuidado humano de ayuda-confianza".

\begin{tabular}{lrrrr}
\hline Factor institucional & \multicolumn{1}{c}{ Siempre } & \multicolumn{1}{c}{ A veces } & \multicolumn{1}{c}{ Nunca } & \multicolumn{1}{c}{ TOTAL } \\
\hline Infraestrutura física & $16(45,7 \%)$ & $18(51,4 \%)$ & $1(2,9 \%)$ & $35(100 \%)$ \\
Condición laboral & $13(37,1 \%)$ & $7(20 \%)$ & $15(42,9 \%)$ & $35(100 \%)$ \\
Horario de trabajo & $15(42,9 \%)$ & $18(51,4 \%)$ & $2(5,7 \%)$ & $35(100 \%)$ \\
Paciente a cargo durante el turno & $13(37,1 \%)$ & $2(5,7 \%)$ & $20(57,2 \%)$ & $35(100 \%)$ \\
Grado de dependencia & $8(22,9 \%)$ & $24(68,6 \%)$ & $3(8,5 \%)$ & $35(100 \%)$ \\
Dotación de personal & $15(42,9 \%)$ & $5(14,2 \%)$ & $15(42,9 \%)$ & $35(100 \%)$ \\
Reconocimiento laboral & $24(68,6)$ & $3(8,5 \%)$ & $8(22,9 \%)$ & $35(100 \%)$ \\
Capacitación & $28(80 \%)$ & $1(2,9 \%)$ & $6(17,1 \%)$ & $35(100 \%)$ \\
\hline
\end{tabular}


Tabla 3. Dimensiones observadas del "Cuidado humano de ayuda-confianza".

\begin{tabular}{lrr}
\hline Cuidado humano & Número & \% \\
\hline Coherencia & 4 & 11,43 \\
$\mathrm{No}$ & 31 & 88,57 \\
$\mathrm{Si}$ & & \\
Empatia & 8 & 22,86 \\
$\mathrm{No}$ & 27 & 77,14 \\
$\mathrm{Si}$ & & \\
Acogida no posesiva & 4 & 11,43 \\
No & 31 & 88,57 \\
$\mathrm{Si}$ & & \\
Comunicación eficaz & 1 & 2,86 \\
No & 34 & 97,14 \\
Si & & \\
\hline
\end{tabular}

En cuanto al factor institucional, son aquellos elementos relacionados con el proceso administrativo y de organización de la Institución, vienen a ser parte de un conjunto de normas y/o estatutos que norman la vida institucional y que impactarán de manera positiva o negativa en los resultados de los objetivos programados, lo que finalmente redundará negativa o positivamente en la imagen institucional (6).

Respecto a la frecuencia del factor institucional para el desarrollo del "Cuidado humano de ayuda-confianza" (tabla 2), se obtuvo para el estudio que un $80 \%$ de los enfermeros consideran que este factor está basado en la capacitación y que el reconocimiento laboral se encuentra presente en un $68,6 \%$ para que se dé un buen cuidado humano; siendo estos resultados importantes ya que a través de la capacitación continua, los enfermeros se van desarrollando, fortaleciendo sus habilidades y destrezas; así mismo, el contar con enfermeros reconocidos permite un mejor desempeño profesional. Estos datos son similares con los resultados hallados en el estudio "Factores que influyen en el profesional de enfermería para la aplicación de los modelos y teorías de enfermería en el cuidado del usuario realizado por Armas, en la Microred de Chosica y Chaclacayo, Lima, donde se obtuvo que estos factores superan el $70 \%$, resaltando que no cuentan con un plan de capacitación, ni cursos sobre temas de modelos y teorías de enfermería, no brinda facilidades para asistir a cursos, no exigen la réplica de los cursos recibidos, no cuentan con condiciones físicas y considera que la elevada demanda no favorece la aplicación de los modelos y teorías, lo cual intervienen o repercuten en la calidad de atención al usuario (6).

De igual manera, el trabajo de Ayala y col., "Evidencias para la filosofía de Watson", tuvo como finalidad evaluar y validar el papel crítico del cuidado en la atención de las personas y su influencia en mejores resultados de la práctica, como una herramienta que evalúa el cuidado de calidad y demanda para la enfermería, condiciones de trabajo que permitan desarrollar el cuidado como un bien social (7).

Finalmente, Abades, en su trabajo "Los cuidados enfermeros en los centros geriátricos según el modelo de Watson", concluyó que es necesario que se planteen nuevos modelos de funcionamiento y de trabajo profesional, los cuales incidirán en el incremento de la eficiencia de la calidad y de los cuidados enfermeros (8). Por tanto las instituciones hospitalarias tienen la responsabilidad de modificar el cuidado de enfermería en las instituciones de salud, para ello debe generar proyectos, programas, procesos de cambio con la finalidad de brindar un cuidado humano, con calidad en la atención.

En cuanto, a las dimensiones del desarrollo del "Cuidado humano de ayuda-confianza", son imprescindibles para un cuidado humano holístico la existencia de coherencia, empatía, acogida no posesiva y comunicación eficaz en el actuar enfermero se encontró que estas dimensiones se encuentran en un alto porcentaje 
(88,57\%, 77,14\%, 87,57\% y 97,14\% respectivamente) y esta importante presencia en el desempeño del profesional enfermero durante el cuidado de los pacientes hace que el cuidado humano se dé en forma eficaz.

Por lo tanto, cabe resaltar que en la aplicación de las dimensiones del "Cuidado humano de ayuda-confianza"; para la coherencia, se obtuvo que un $91,40 \%$ de los enfermeros muestra en su actuar ser coherentes para la relación del cuidado humano, mostrando una conducta correcta en todo momento, cumpliendo con eficacia su deber.

Mientras para la empatía, se evidenció que 97,1\% de los enfermeros observados durante el cuidado de los pacientes reflejan en sus intervenciones de enfermería, preguntas sino entiende lo que comunica el paciente, favoreciendo la relación de cuidado humano de ayudaconfianza enfermera-paciente. Por lo tanto; el ser empático llevará como enfermeros a tener la capacidad de poder comprender lo que experimenta nuestro paciente para poder ayudarlo en su cuidado considerando sus sentimientos y entendimiento.

Asi mismo, para la acogida no posesiva, se obtuvo que un $100 \%$ de los enfermeros observados muestran relación del lenguaje corporal con la comunicación, muestran contacto visual y de escucha ante las necesidades del paciente, ponen límites, centran la comunicación evitando aumento de la ansiedad; siendo todo ellos esencial para que se pueda establecer una verdadera relación de ayuda, logrando en el paciente la confianza en el profesional enfermero para favorecer su cuidado.

Finalmente para la comunicación eficaz, se obtuvo que un $100 \%$ de los enfermeros se comunican con claridad y precisión; existiendo una comunicación eficaz para el desarrollo del cuidado humano. Esta comunicación eficaz tiene componentes de respuestas cognitivas, afectuosas y conductuales, mostrando los enfermeros capacidad para expresarse claramente y de manera concreta para ser comprendidos; así mismo, expresa con la comunicación sus sentimientos respecto a lo que está diciendo y necesitando.

Estos resultados son similares al estudio realizado por Do Nacimento y col., en su estudio "Comprender las dimensiones de los cuidados intensivos: la teoría del cuidado transpersonal y complejo", se enfocan en el cuidado como una relación de ayuda y con toque afectivo, concluye asumir actitudes diferentes frente al cuidado y a las personas, con un cuidado humano con sensibilidad, empatía y satisfacción (9).

Así mismo, Martins y col., en su estudio "Resignificando la humanización desde el cuidado en el curso del vivir humano", proponen que el cuidado debe estar basado en la comunicación, escucha, aproximación del equipo con usuarios y familiares y reconociendo la singularidad del otro por el dialogo (10).

Igualmente, Rivera y col., en su estudio "Cuidado Humanizado de enfermería: Visibilizando la teoría y la investigación en la práctica, en la clínica del Country", manifiestan que las categorías del cuidado percibidos fueron: priorizar a la persona $(89,8 \%)$, apoyo emocional $(79,6 \%)$, la empatía $(77,7 \%)$ y la disponibilidad para la atención $(85,4 \%)(9)$.

Los mencionados estudios confirman que es imprescindible la aplicación de las dimensiones del cuarto factor "Cuidado humano de ayuda-confianza", de la Teoría de Jean Watson durante el cuidado, para lograr así un cuidado holístico, individualizado y humano, no dejando relegado acciones como la comunicación eficaz y el interactuar con el paciente y familia en forma cercana. Finalmente, éste estudio acerca de la aplicación del "Cuidado humano de ayuda confianza", de la teoría de Jean Watson, tuvo algunas limitaciones en cuanto al tiempo para la aplicación de la guía de observación, ya que todo la población de estudio se desempeña en una misma área física, por ellos se tuvo que realizar la observación por grupos de guardia y en días diferentes, contando al final con solo dos horas de observación para cada enfermero, además el estar constantemente en el servicio podría alertar a los evaluados creando sesgos que podrían alterar los resultados.

\section{REFERENCIAS BIBLIOGRAFICAS}

1. Gallardo K. La comunicación: llave maestra para la aplicación de valores humanísticos y altruistas en el cuidado de niños. Revista Cultura del Cuidado Enfermería. 2011;8(1): 5-16.

2. Beltrán O. Factores que influyen en la interacción humana del cuidado de Enfermería. Invest Educ enferm. 2006; 24 (2): 144-150.

3. Orozco L. Relación enfermera-paciente. En: Hernández JL. Bioética General. Ciudad de México: Manual Moderno; 2002.

4. Watson J. Human caring science: A theory of nursing. Second edition. Burlington: Jones - Bartlett Learning; 2012.

5. Raile M, Marriner A. Modelos y teorías en enfermería. Séptima edición. Barcelona: Elsevier España; 2011. 
6. Armas E. Factores que influyen en el profesional de Enfermería para la aplicación de los modelos y teorías de enfermería en el cuidado del usuario de los establecimientos de salud de la MICRORED de Chosica I y Chaclacayo. Lima: Universidad Nacional Mayor de San Marcos; 2007.

7. Ayala-Valenzuela R, Calvo-Gil M; Torres-Andrade M, Koch-Ewertz T. Evidencias para la Filosofía de Watson: versión preliminar del Caring Behaviors Assessment en Chile. Revista Cubana de Enfermería 2010;26(1):42-51.

8. Abades M. Los cuidados enfermeros en los centros geriátricos según el modelo de Watson. Ver Gerokomos. 2007; 18(4): 176-180.
9. Rivera T. Cuidado humanizado de enfermería: Visibilizando la teoría y la investigación en la práctica, en la clínica del Country. Rev Actual Enfer. 2007; 10(4): 15-21.

10. Martins J, Stein D, Da Silva R, Lorenzini A, De Albuquerque G. Resignificando la humanización desde el cuidado en el curso de vivir humano. Rev Enferm UERJ. 2008;16(2): 276-81.

\section{Correspondencia:}

Corolina Sebastián Llancari

Correo electrónico: carolina.sebastian@upch.pe

Fecha de Recepción: 07 de abril del 2016.

Fecha de aceptación: 31 de mayo del 2016. 